\title{
Embolización arterial en epistaxis
}

\author{
Artery Embolization in epistaxis
}

Cristián González $\mathrm{G}^{1}$, Martin Einersen $A^{2}$, Mario Gutiérrez $A^{3}$.

\begin{abstract}
RESUMEN
La epistaxis constituye una emergencia frecuente en otorrinolaringología. Diariamente se presentan, en servicios de urgencia de hospitales y clínicas, pacientes portadores de hemorragia nasal asociadas o secundarias a hipertensión arterial, traumatismos faciales 0 procesos tumorales de la región facial. Generalmente, en una gran parte de ellas el taponamiento anterior y/o posterior constituye la terapia más efectiva y la hemorragia se controla rápidamente. Sin embargo, hay pacientes en los que por las características de su cuadro clínico o por lesiones vasculares traumáticas, requieren de un manejo más agresivo para controlar definitivamente esta situación de urgencia.

La embolización arterial de la arteria maxilar interna constituye una alternativa eficaz para controlar, en forma definitiva, las hemorragias nasales recurrentes o incohercibles.

Dada la particular irrigación de la región facial, que permite acceder a una gran cantidad de circulación colateral es posible efectuar este procedimiento sin riesgos para la irrigación facial, que requiere del apoyo y soporte de la imagenología invasiva en manos de un operador experimentado.

Se describen tres casos clínicos de pacientes con epistaxis severa, portadores de traumatismos por proyectil y tumor de seno maxilar, que requirieron como alternativa final la embolización de la arteria maxilar interna Se describe la técnica y los resultados obtenidos.

Palabras claves: Epistaxis, Embolización arterial.
\end{abstract}

\section{SUMMARY}

Epistaxis constitutes a frequent emergency in otolaryngology. At emergency wards in hospitals and clinics there are patients daily suffering from nasal hemorrhage associated or secondary to arterial hypertension, facial traumatisms or tumoral processes of the facial region. Generally, in great part of them, the anterior and/or posterior obturation is the most effective therapy and the hemorrage is quickly controlled. However, there are patients who due to the characteristics of their clinical situation or traumatic vascular damages, require a more aggressive handling to control in a definite way this urgency situation.

\footnotetext{
${ }^{1}$ Médico Residente, Servicio de Otorrinolaringología, Hospital Guillermo Grant Benavente.

${ }^{2}$ Médico Radiólogo, Servicio de Radiología, Hospital Guillermo Grant Benavente.

${ }^{3}$ Grujano Máxilo Facial, Servicio de Otorrinolaringología, Hospital Guillermo Grant Benavente.
} 
Arterial embolization of the inner maxillary artery constitutes an efficient alternative to control in a definite way the recurrent or incoercible nasal hemorrages.

Given the particular irrigation of the facial region, that allows access to a big amount of collateral circulation, it is possible to apply this procedure without risks to the facial irrigation, which requires the support of invasive imagery in the hands of an experimented operator.

Three clinical cases are described, of patients with severe epistaxis, with trauma due to projectile and tumor in the maxillary cavity, who required as a final alternative, the embolization of the inner maxillary artery. The technique and results obtained are described.

Key words: Epistaxis, Arterial embolization.

\section{INTRODUCCIÓN}

La frecuencia de la epistaxis es de $60 \%$ en la población general y aproximadamente un $6 \%$ de éstas requieren tratamiento médico ${ }^{1}$, ya sea tapona miento anterior, taponamiento posterior, cauteriza ción o métodos más invasivos como la embolización, lo cual pone a pruebala interacción entre el otorrino y el radiólogo con el objetivo de solucionar un problema que pone en peligro la vida de un enfermo.

La epistaxis de difícil manejo que ha requerido taponamiento posterior y cuyo resultado no es el esperado, puede ser manejada con otras técnicas como la embolización arterial, constituyendo unasitua ción en la que los conocimientos y destrezas del otorrinolaringólogo y el radiólogo intervencionistapue den complementarse y terminar con la emergencia

La epistaxis puede ser primaria o secundaria a traumatismo, malformaciones arteriovenosas, coagulopatías o tumores como el angiofibroma juvenil o de senos paranasales ${ }^{2,3}$. Puede ser de localización anterior o posterior y requerir taponamiento anterior o posterior, cauterización endoscópica o ligadura arterial.

La embolización terapéutica consiste en depositar en el lumen arterial, un material destinado a provocar su obstrucción. Para obtener éxito en este procedimiento se requiere de un radiólogo intervencionista, con acabados conocimientos de anatomía vascular y que esté familiarizado con las técnicas de cateterismo y punción.
Además, se debe contar con un lugar adecuado, un buen equipamiento radiológico, equipos de monitoreo, implementos de reanimación y personal entrenado en el manejo de reacciones adversas.

La encuesta preintervención ${ }^{2}$ es importante en relación a conocer antecedentes alérgicos y preve nir reacciones adversas a medios de contraste. Se debe contar con el consentimiento informado, dar a conocer información acerca de la anestesia que recibirá el paciente y los cuidados postoperatorios 4 .

Scroop y colaboradores ${ }^{2}$, en 2003, presentaron 119 casos de epistaxis refractaria a taponamiento y cauterización tratadas con embolización arterial, obteniendo un $100 \%$ de éxito en el manejo de la hemorragia, al embolizar las arterias maxilares internas en forma bilateral y facial del lado sangrante, y un $87 \%$ embolizando sólo la arteria maxilar interna del lado sangrante, sin presentar ningún tipo de complicaciones.

Oguni y colaboradores ${ }^{1}$, demuestran en 37 casos de epistaxis refractaria, un $94,6 \%$ de éxito con dos recurrencias a los siete días, una de las cuales se resolvió médicamente y la otra con tratamiento quirúrgico y tres embolizaciones más, describiendo un $45 \%$ de complicaciones leves como dolor facial y cefalea

Entre las complicaciones ${ }^{6}$ severas del procedimiento se describen: accidente vascular encefálico, ceguera, necrosis cutánea, parálisis facial en un 2 a $3 \%$ y complicaciones menores como cefalea y dolor facial en un $30 \%$. 
日 objetivo de esta publicación es presentar nuestra experiencia, en la embolización arterial para solucionar epistaxis, en pacientes portadores de lesiones traumáticas y tumorales malignas en los servicios de Atorrinolaringología y Radiología del Hospital Regional de Concepción entre agosto de 2003 y marzo de 2004; destacando la importancia de conocer y manejar como alternativa, esta técnica para el efectivo control de la epistaxis.

\section{MATERIAL Y MÉTODO}

Se revisan tres casos clínicos de epistaxis. Dos de ellos por herida con arma de fuego en la región nasogeniana y otro portador de un tumor del seno maxilar derecho.

日 primer paciente, de 35 años fue herido con arma de fuego calibre 38, munición de alta velocidad y capacidad de cavitación. $\mathrm{日}$ proyectil penetró en la región nasogeniana, describiendo un trayecto que pasa por el piso de la pared lateral de la fosa nasal derecha y se localizó en el espacio parafaríngeo derecho. Ingresó en mayo de 2003, al Servicio de Aorrinolaringología con una severa epistaxis que requirió el uso de taponamiento posterior. Se intentó extracción del proyectil lo que no se logra, evolucionando en el postoperatorio con intenso dolor facial y profusa epistaxis, por lo que se plantea embolizar, cateterizándose la arteria carótida externa derecha, bajo sedación y en pabellón de hemodinamia Se logra visualizar angiográficamente extravasación del medio de contraste de la arteria maxilar interna distal. Paralelamente se realiza estudio angiográfico de la arteria maxilar contralateral sin que se aprecie extravasación del medio de contraste. De inmediato se procede a embolizar con partículas de gelita, lográndose oclusión completa de la arteria maxilar interna derecha $\mathrm{日}$ estudio de la arteria carótida externa izquierda no mostró anomalías vasculares.

日 segundo paciente, de 20 años, fue herido abala en laregión nasal derechacon armacalibre22 en enero de 2004, extrayéndose el proyectil por vía endoscópica en el Hospital Las Higueras de Talcahuano, evolucionando con epistaxis que no cedió con taponamiento posterior. Fuederivado al Hospital Regional de Concepción, donde se realizó angiografía de la arteria carótida externa derecha que mostró extravasación del medio de contraste de la arteria maxilar interna a distal. Se procedió a embolizar la arteria maxilar interna derecha cediendo por completo la epistaxis. $\mathrm{\theta}$ estudio de la arteria carótida externa izquierda fue normal.

日 tercer paciente corresponde a un hombre de 65 años, portador de un carcinoma epidermoide de seno maxilar derecho, que recibió radioterapia y quimioterapia en enero de 2002 y que reingresó por recidiva tumoral y epistaxis en marzo de 2004. Dada la cuantía de la hemorragia, que comprometió hemodimámicamente al paciente, se realizó de inmediato angiografía de arteria carótida externa e interna derechas, demostrándose extravasación activa de arteria maxilar interna, embolizándose con gelita la misma arteria, procedimiento que resultó exitoso, sin recidiva de la hemorragia $\theta$ estudio de la arteria carótida externa izquierda resultó normal.

\section{RESULTADOS}

En los tres casos reportados se logró cohibir completamente la hemorragia nasal. En el seguimiento de los tres pacientes, hasta el momento, no ha habido recidiva de la epistaxis. Tampoco se presentaron complicaciones mayores; siendo el algia facial, la única complicación menor presentándose en un paciente solamente.

\section{DISCUSIÓN}

La epistaxis constituye una patología frecuente en la población general, que requiere una rápida y acertada intervención del médico otorrinolaringólogo para su resolución adecuada.

En epistaxis refractaria a taponamiento anterior, cauterización endoscópica o taponamiento posterior, la embolización percutánea de la arteria sangrante constituye una excelente alternativa para terminar con el sangrado, requiriendo laacción de un equipo radiológico intervencionista para dar solución al problema

Por otro lado, el tratamiento quirúrgico implica la ligadura de la arteria maxilar interna y sus ramas. Sin embargo, este tratamiento tiene una tasa de fracaso de entre un 15 a un $22 \%$, debido a ligadura incompleta o a existencia de arterias colaterales ${ }^{6}$. Desde 1974 
cuando Sokoloff y cols ${ }^{7}$ describieron la técnica de embolización para el manejo de las epistaxis intrata bles, ésta ha tenido cada ves más aceptación. La embolización, con partículas de la arteria maxilar internay si es necesario la arteria facial, tiene un éxito de entre un 82 al 97\% 8,9 en la terapia de la epistaxis.

Complicaciones como necrosis distal, cicatrices, ceguera y parálisis facial han sido descritas para esta técnica ${ }^{10}$, pero en general las complicaciones son poco frecuentes y la incidencia reportada en la literatura es de entre un 0,1 al $3 \%{ }^{11,12}$. 日 factor más importanteen estos casos es la existencia de anastomosis peligrosas de la carótida externa con la carótida interna o la arteria oftámica y por otro lado, el uso de partículas muy pequeñas, las que tienen más riesgos de producir necrosis distal y atravesar anastomosis peligrosas. uso de partículas muy gruesas provocarían oclusiones muy proximales y poco efectivas, además detener más posibilidades de refluir hacia otros territorios vasculares no deseados ${ }^{11}$. En general, el rango de tamaño de las partículas habitualmente utilizado en estas terapias es de 150 a $500 \mu \mathrm{m}^{4}$. Ara complicación posible es la aspiración de sangre acumulada en el rinofarinx durante el procedimiento. Aun en pacientes con taponamiento nasal la posición supina, en que se realiza la terapia, puede favorecer esta complicación. Sin embargo en la mayoría de los casos la embolización requiere anestesia general con protección de la vía aérea

Usualmente la embolización se realiza avanzando un catéter diagnóstico o un microcatéter hasta la arteria maxilar internaatravés del cual se introducen micropartículas de polivinil-alcohol de entre 150 a $500 \mu \mathrm{m}$ o gelita. Las arterias más involucradas en las epistaxis espontáneas son las esfenopalatinas y la gran palatina En nuestra experiencia, el procedimiento de embolización percutánea de la arteria proximal a la rama sangrante, soluciona el problema y concuerda con lo señalado en la literatura ${ }^{10}$.

Constituye por lo tanto un método que, realizado por un médico radiólogo intervencionista de experiencia, tiene un éxito cercano al $100 \%$ en cohibir una hemorragia de magnitud tal, que podría poner en peligro la vida de un enfermo, siendo un método seguro, de baja morbilidad y que puede repetirse de ser necesario.

\section{BIBLIOGRAFÍA}

1. OGUNI, Yasunaga T. Superselective Embolization for Intractable Epistaxis. Br J Radiol 2000; 73 (875): 1148-53.

2. SCROOP R, VENTURINI C, SONG J. Endovascular Treatment of Epistaxis. Seminars in Interventional Radiology 2000; 20 (1): 51-7.

3. ORTIZ JM, BHATTACHARRYYA N. Management Pitfalls in the Use of Embolization for the Treatment of Severe Epistaxis. Ear Nose Throat $J$ 2002; 81 (3): 178-83.

4. KOBBE CJ, HOROMTZM. Endovascular Particulate and Alcohol Embolization for Near-Fatal Epistaxis from Skull Base Vascular Malformation. Pediatr Neurosurg 2001; 35 (5): 257-61.

5. MoRRISP. Interventional and Endovascular Thera py of Nervous System. New York, Springer 2002; 259-66.

6. MontGomerY WW, REARDON EJ. Controversy in Otolaryngology. Philadelphia, J.B. 1980 Saunders 315-9.

7. SokáfF J Y cols. Therapeutic percutaneous embolization in intractable epistaxis. Radiology 1974; 111: 285-7.

8. PARNES LS Y cols. Percutaneous embolization for control of nasal blood circulation. Laryngoscope 1987; 97: 1312-15.

9. SINILUOTO TMJ Y cols. Embolization for the treatment of posterior epistaxis. An analysis of 31 cases. Arch Otoloryngol Head Neck Surg 1993; 119: 837-41.

10. MOREAU S Y COLs. Supraselective embolization in intractable epistaxis: review of 45 cases. Laryngoscope 1998; 108: 798-888.

11. VITEK JJ. Idiopathic intractable epistaxis: endovascular therapy. Radiology 1991; 181: 113-16.

12. STRUTZ J Y cols. Uncontrollable epistaxis. Angiografic localization and embolization. Arch Otoloryngol Head Neck Surg 1990; 116: 697-9. 